\title{
A Novel Consumer Purchase Behavior Recognition Method Using Ensemble Learning Algorithm
}

\author{
Peng Wang $(\mathbb{D}$ and Zhengliang Xu \\ School of Management, Jilin University, Changchun 130021, China \\ Correspondence should be addressed to Peng Wang; wangpeng11@mails.jlu.edu.cn
}

Received 19 November 2020; Revised 2 December 2020; Accepted 4 December 2020; Published 21 December 2020

Academic Editor: Yi-Zhang Jiang

Copyright (c) 2020 Peng Wang and Zhengliang Xu. This is an open access article distributed under the Creative Commons Attribution License, which permits unrestricted use, distribution, and reproduction in any medium, provided the original work is properly cited.

\begin{abstract}
With the prosperous development of e-commerce platforms, consumer returns often occur. The issue of returns has become a stumbling block to the profitability of e-commerce companies. To protect consumers' purchase rights, the Chinese government has introduced a 7-day unreasonable return policy. In order to use the return policy to attract consumers to buy, various e-commerce platforms have created a more relaxed and convenient return environment for consumers. On the one hand, the introduction of the return policy has increased customer trust in e-commerce platforms and stimulated purchase demand. On the other hand, the return behavior also increases the cost of the e-commerce platform. With the upgrading of consumption, customers pay more attention to personalized experience. In addition to considering price when purchasing online, the quality of services provided by e-commerce platforms will also directly affect customers' purchasing decisions and return behavior. Therefore, under the personalized return policy of the e-commerce platform, whether consumers will make another purchase is worth studying. In order to achieve this goal, an ensemble learning method (AdaBoost-FSVM) based on fuzzy support vector machine (FSVM) is applied to predict the purchase intention of consumers. First, the grid search method is used to optimize the modeling parameters of the FSVM base classifier. Second, the AdaBoost-FSVM ensemble prediction model is constructed by using multiple base classifiers. In order to evaluate the performance of the prediction models used, logistic regression (LR), support vector machine (SVM), FSVM, random forest (RF), and XGBoost were used to construct prediction models for purchasing behavior. The experimental results demonstrate that the method used in this study has a more accurate prediction effect than the comparison algorithms. The predictive model used in this study can be used in the recommendation system of shopping websites and can also be used to guide e-commerce companies to customize various preferential policies and services, so as to quickly and accurately stimulate the purchase intention of more potential consumers.
\end{abstract}

\section{Introduction}

The development of the Internet has promoted the birth of various network platforms, and e-commerce has become a part of people's lives. Online shopping has many advantages over physical store shopping. For example, consumers can purchase goods at any time and place without being restricted by time and space. At the same time, online shopping provides a broader market for both parties. Sellers can sell to consumers around the world, and buyers can choose products from any store in the world. E-commerce also reduces the intermediate links of commodity circulation, thereby greatly reducing the cost of commodity circulation and transactions. Online shopping also promotes a personalized shopping process. Consumers can filter the price, evaluation, service, and other parameters of commodities according to their needs, so as to lock the items they like. Online shopping has become an indispensable part of people's lives. Consumers are important participants in e-commerce, and the influencing factors of their purchase behavior are crucial to promoting transactions and the sustainable development of e-commerce platforms.

To protect consumers' purchase rights, the government has introduced a 7-day unreasonable return policy. Under the government's return policy framework, various e-commerce platforms have successively introduced their 
own return policies and services. The impact of the return policy on the purchase behavior of consumers has become a subject of research by many scholars. Reference [1] studied the impact of full-refund and partial-refund policies on consumers' purchase intentions. Reference [2] constructed a profit maximization model to study the relationship between sales volume and return volume and obtained the optimal refund policy and product price. Reference [3] constructed a model based on consumer preferences to derive the seller's optimal pricing and return fee policy. Reference [4] built a model to analyze full-refund sum, partial-refund policy, and pricing strategy by introducing the return policy into the supply chain contract. Reference [5] built several theoretical models to analyze the relationship between return policy, product quality, and product pricing based on consumer purchase and return behavior. The experimental results show that the gift policy and the return policy are mutually influencing and complementary. Reference [6] derives the optimal pricing and refund policy of fashion product retailers. In their research, both full-refund and partial-refund policies were incorporated into the model. There is a similar study on the return policy of fashion fast-selling products in [7]. After the research of [8], [9] explored the issue of return service fees for mass-customized fashion products and deduced under what circumstances e-commerce companies can provide free returns for better results. Reference [10] uses an empirical method to study the impact of return strategies on customer purchases. It divides online purchase behavior into two stages. Due to the asymmetry of information, customers face greater risk of return in the second stage. Through three sets of online purchase experiments, the relationship among the looseness of return, customer purchase decision, and merchant profit is verified. Reference [11] believes that customer return is an inevitable behavior, but it is not absolutely a bad thing for companies. The econometric model is used to demonstrate the behavior of returns during the transaction and the impact of returns on the future decisions of enterprises and consumers. The research finally demonstrated the role of returns in the transaction process. Reference [12] studies the impact of return behavior from another perspective. If e-commerce companies can provide return services for customers' customized needs, they can gain huge advantages. From the tools used in these studies, it can be found that machine learning algorithms [13-21] are widely used to predict purchase behavior.

Most of the above works study the impact of the return policy provided by e-commerce companies on the purchasing power of consumers under the unreasonable refund policy. These studies also have the following problems: One is that they did not take into account the return policies of retailers in all e-commerce platforms. Second, they did not pay attention to the return services provided by various merchants. Third, the economic and labor costs of returning goods are not included in the calculation. In response to these problems, this article focuses on the return policies of existing e-commerce companies and uses a new ensemble learning method to predict consumers' buying behavior. By analyzing user behavior in historical data and modeling, whether the user will buy the product again next time can be predicted. From the prediction results, what kind of return policy and return service can improve the purchasing power of consumers can be analyzed. The contributions of this study mainly include the four following points:

(1) Since the collected original data source is not complete, there are missing values and data duplication, inconsistent attribute data types, and noise effects. This study first carried out relevant preprocessing on the data.

(2) Through the analysis of data and consumer purchasing behavior, the basic characteristics related to the research question are found out; and, according to the different situations of each data set, the features are associated and extracted.

(3) The AdaBoost-FSVM ensemble learning method is used to predict consumers' buying behavior. The ensemble method uses AdaBoost algorithm, and FSVM is used as a weak classifier. FSVM introduces a membership mechanism on the basis of SVM, so that the classifier has better noise resistance. Comparative experiments show that the ensemble learning method used in this paper has better prediction effects than other nonensemble methods and XGBoost ensemble methods.

(4) Through the analysis of the experimental results, the relationship between the return policy of the e-commerce company, the return service, and the purchasing power of consumers is obtained. This connection can guide e-commerce companies on how to optimize return policies and services, so as to achieve the best sales relationship between e-commerce companies and consumers.

\section{Related Information}

2.1. E-Commerce Company's Return Policy. In 2014, the government revised the Consumer Protection Law. This law formally introduced a policy of returning goods for online shopping, which is "7 days without reason." This policy stipulates that consumers have the right to return the goods within 7 days from the date of receipt of the goods without giving reasons. On the one hand, the unreasonable return policy can protect the legitimate rights and interests of consumers. On the other hand, providing an unreasonable return policy is also an effective means for major e-commerce platforms to attract consumers and stimulate purchase demand. Consumers not only consider factors related to the product itself when shopping online but also value the level of service provided by the seller. The return policy is an important measure. The current return policies of several mainstream e-commerce platforms are shown in Table 1.

By comparing the return policies of major e-commerce companies, the following points can be analyzed:

(1) All e-commerce platforms promise to return goods without reason within 7 days, but the specific return policies and services are different. 
TABLE 1: Return policies of major e-commerce platforms.

\begin{tabular}{|c|c|c|c|c|}
\hline $\begin{array}{l}\text { E-commerce } \\
\text { platforms }\end{array}$ & Type & Return policy & $\begin{array}{l}\text { Return } \\
\text { amount }\end{array}$ & Logistics costs \\
\hline TMALL.COM & $\mathrm{B} 2 \mathrm{C}$ & 7 days no reason to return & Full refund & $\begin{array}{c}\text { Nonquality issues are borne by the } \\
\text { customer }\end{array}$ \\
\hline TAOBAO & $\mathrm{C} 2 \mathrm{C}$ & 7 days no reason to return & Full refund & $\begin{array}{c}\text { Nonquality issues are borne by the } \\
\text { customer }\end{array}$ \\
\hline JD.COM & $\begin{array}{l}\text { Self-operated + platform e- } \\
\text { commerce }\end{array}$ & 7 days no reason to return & Full refund & $\begin{array}{c}\text { Nonquality issues are borne by the } \\
\text { customer }\end{array}$ \\
\hline VIP.COM & Self-operated & 7 days no reason to return & Full refund & $\begin{array}{c}\text { Nonquality issues are borne by the } \\
\text { customer }\end{array}$ \\
\hline SUNING.COM & $\begin{array}{l}\text { Self-operated + platform e- } \\
\text { commerce }\end{array}$ & 7 days no reason to return & Full refund & $\begin{array}{l}\text { Nonquality issues are borne by the } \\
\text { customer }\end{array}$ \\
\hline JUMEI.COM & Vertical e-commerce & $\begin{array}{c}30 \text { days no reason to } \\
\text { return }\end{array}$ & Full refund & $\begin{array}{c}\text { Platform subsidies } 10 \text { yuan for } \\
\text { shipping }\end{array}$ \\
\hline AMAZON.CN & $\begin{array}{l}\text { Self-operated + platform e- } \\
\text { commerce }\end{array}$ & $\begin{array}{l}7 / 15 / 30 \text { days no reason to } \\
\text { return }\end{array}$ & Full refund & $\begin{array}{l}\text { Nonquality issues are borne by the } \\
\text { customer }\end{array}$ \\
\hline
\end{tabular}

(2) Vertical e-commerce companies have a more relaxed return policy than comprehensive e-commerce companies. For example, JUMEI.COM promises to return goods without reason within 30 days. Even used goods can be returned, and the postage paid by consumers will be subsidized. Comprehensive e-commerce companies such as TMALL.COM and JD.COM have stricter return policies and will not subsidize postage.

(3) Ensemble e-commerce companies provide more considerate return services than vertical e-commerce companies. For example, JD.COM will require retailers to respond to customers' 7-day unreasonable return application within 24 hours. If the consumer needs to pick up the goods, the staff must pick it up within 48 hours. Retailers should give clear advice on postsale disposal within 48 hours after receiving the message of the returned goods from consumers.

2.2. Influencing Factors of Consumers' Online Buying Behavior. In addition to considering product price factors when consumers buy online, the retailer's service level will also directly affect consumer demand and return behavior. Therefore, in addition to product competition and price competition, service competition is also inevitable. All major e-commerce platforms use various means to improve service levels, bring consumers a better shopping experience, and reduce the possibility of mismatch between customer expectations and actual products. In 2017, the China Consumers Association conducted a survey, the subject of which was "online shopping integrity and consumer awareness." The results of the survey give the factors that affect consumers' online purchases. The details are shown in Figure 1.

From the results shown in Figure 1, the fact that consumers will be affected by many factors when shopping online can be analyzed. $70 \%$ of customers will pay attention to "product/service quality," and $60 \%$ of customers will pay attention to "product/service price." It can be seen that the key factor of service quality as a means for enterprises to enhance competitiveness and attract consumers is crucial.

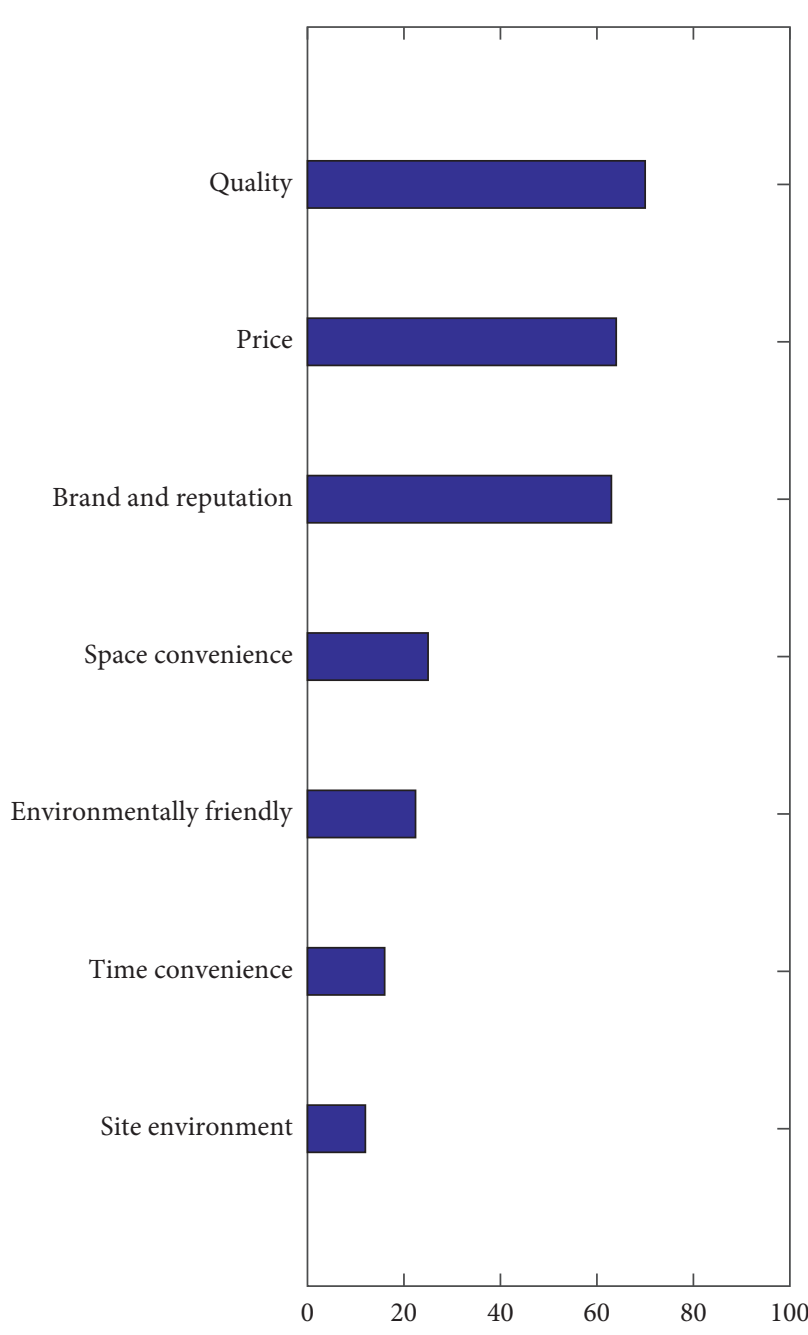

FIGURE 1: Influencing factors of consumers' online purchase.

2.3. Consumer Repurchase Behavior Analysis. In real life, consumers use online shopping platforms to screen, collect, and add to shopping carts. These behaviors reflect the customer's preference for the target product. The shopping groups include young people, old people, office workers, and 
school students. Different people have different preferences. For example, young people are keen to purchase electronic products, the elderly like to purchase daily household items or food materials, and school students like to purchase school supplies and snack foods. Therefore, from the perspective of consumers, some consumers may often repeatedly purchase a certain type of goods, such as daily vegetables and fruits, with a high repeat purchase rate. However, some consumers only occasionally place orders for electronic products and so forth, and the repeat purchase rate is low. There are also consumers who are keen on shopping, purchasing a wide variety of items, and placing orders frequently. However, due to the complexity of the product categories, some products may have a high repetition rate and others may have a low repetition rate.

The goal of this experiment is to select characteristic data by analyzing the results. Ensemble learning algorithms are used to model and predict the probability of consumers buying again. A feasible solution is to make predictions through two classification methods. This study uses supervised learning, and historical big data has provided training samples and target values. Therefore, more attention should be paid to repurchase products when analyzing. For a certain type of consumer, it is important to know which products are often repurchased. It is very meaningful to find out the underlying laws.

2.4. Order Information Analysis. Finding useful information and extracting features from historical data are key steps in prediction. Generally, the fields in each table in the data set are meaningful for predicting reshopping behavior, but there are also a small part of the fields that are mainly used for description and have little meaning. Fields that are helpful for prediction can be converted into valid features through feature extraction. Fields of little significance can be ignored directly. Reorder is an important feature. During the training process, the accuracy of the prediction can be verified. Information such as the time period when the product is purchased plays an important role in predicting the user's reconstruction behavior.

In real life, people's purchasing trends are generally regular. Orders are more concentrated on holidays than on weekdays, and orders are more concentrated during the day than at night. The more high-quality goods are more likely to be repeated purchases, and such goods are often placed in the shopping cart first. More useful information can be analyzed in more detail.

\section{Consumer Repurchase Behavior Prediction}

3.1. Forecasting Process. The construction process of the consumer buying behavior prediction model is shown in Figure 2. First, the feature from the original data to obtain the feature summary table is constructed, and the training set and test set are selected. Second, the features are extracted from the training set. The feature extraction result is to select the features that the prediction algorithm needs to use from the feature list. Third, the training set after feature selection

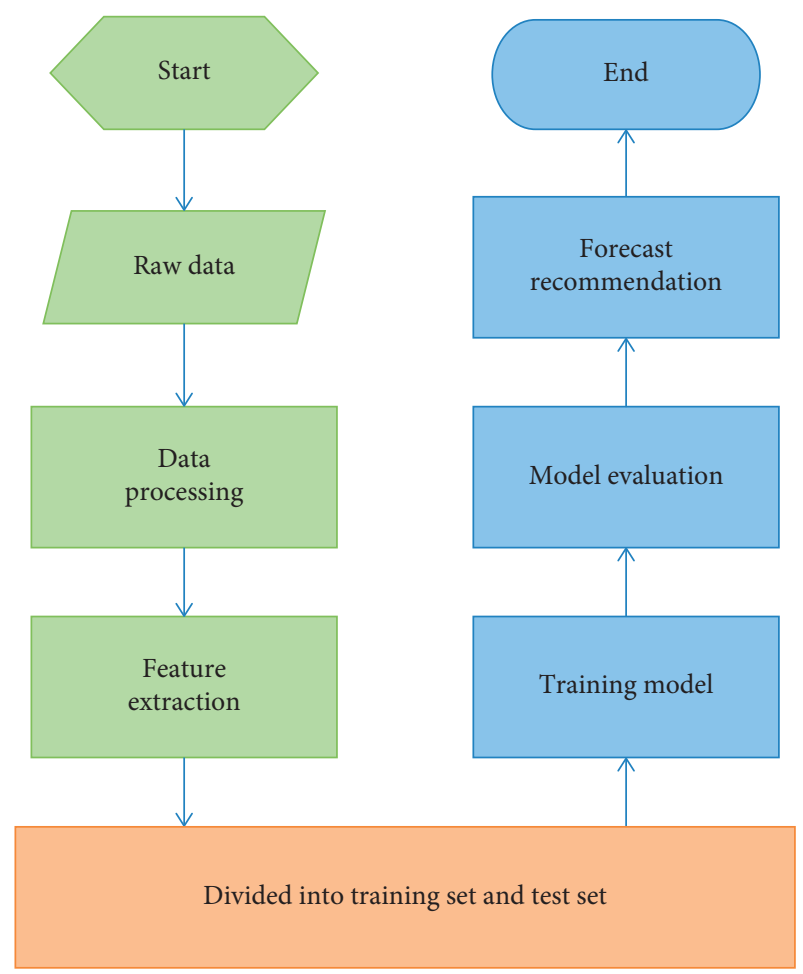

Figure 2: Consumer buying behavior prediction process.

is input into the algorithm for learning to obtain a predictive model. The test set is input into the prediction model to obtain the prediction result.

3.2. Raw Data and Preprocessing. The data set used is data provided by a competition in the Kaggle machine learning competition community. The purpose of the competition is to predict which products consumers will repeat next time based on historical data. The open-source historical sales data of Instacart can be downloaded online. The data structure is shown in Tables 2-6.

3.3. Feature Extraction. The original data has a high dimensionality and contains poor quality features, which leads to a long training time for the model. Effective data features not only will reduce the complexity of modeling but also are very important for improving the accuracy of prediction. Based on the needs of this research, valuable features are selected from the original sample data. The selected features mainly include basic data features, user features, product features, and user product features, totaling 30 . The selected features are shown in Tables 7-10.

3.4. Model Training. In the AdaBoost $[22,23]$ algorithm, the feature weights of each training data sample are initialized, and these weights form vector D. First, a weak classifier is trained through the training data set. The classification error rate of the classifier is also calculated. Then, the next round of weak classifiers is trained by changing the weight of the data set. In the second training of the classifier, the weight of 
TABLE 2: Shelf table.

\begin{tabular}{lccc}
\hline Column name & Type & Description & Example \\
\hline Aisle_id & Integer & Shelf identification number & {$[1: 100]$} \\
Aisle & String & Shelf name & Instant food \\
\hline
\end{tabular}

TABLe 3: Department table.

\begin{tabular}{lccc}
\hline Column name & Type & Description & Example \\
\hline Department_id & Integer & Department ID & {$[1: 21]$} \\
Department & String & Department name & Frozen \\
\hline
\end{tabular}

TABle 4: Product list.

\begin{tabular}{lccc}
\hline Column name & Type & Description & Example \\
\hline Product_id & Integer & Product identification number & {$[1: 21]$} \\
Product & String & Product name & Frozen \\
Aisle_id & Integer & Foreign key & N/A \\
Department_id & String & Foreign key & N/A \\
\hline
\end{tabular}

TABLe 5: Order table.

\begin{tabular}{lccc}
\hline Column name & Type & Description & Example \\
\hline Order_id & Integer & Order identification number & $1,2,3, \ldots$ \\
User_id & Integer & Order identification number & $1,2,3, \ldots$ \\
Eval_set & String & Sample type & [train, test, prior] \\
Department_id & Integer & User order sequence number & $1,2,3, \ldots$ \\
Order_dow & Integer & What day is the order & {$[1: 7]$} \\
Order_hour_of_day & Integer & The specific moment of the order & {$[0: 23]$} \\
\hline
\end{tabular}

TABLE 6: Order product relationship table.

\begin{tabular}{|c|c|c|c|}
\hline Column name & Type & Description & Example \\
\hline Order_id & Integer & Foreign key & $\mathrm{N} / \mathrm{A}$ \\
\hline Product & String & Foreign key & N/A \\
\hline Add_to_cart_order & Integer & The serial number of the goods in the order & $1,2,3, \ldots$ \\
\hline Reordered & Integer & Repeat purchase logo & 1 or 0 \\
\hline
\end{tabular}

TABle 7: Basic characteristics table.

\begin{tabular}{lcc}
\hline Feature variable & Feature name & Remarks \\
\hline User_id & User ID & Fields of the prediction result set \\
Product_id & Product ID & Fields of the prediction result set \\
Order_number & Order ID & Medium importance \\
Add_to_cart_order & Item ID in order & Higher importance \\
Recordered & Repeat purchase logo & Fields of the training data set \\
Order_dow & What day of the week the order was placed & Generally important \\
Order_hour_of_day & Time of order & Generally important \\
Days_since_prior_order & Number of days since the last order & Medium importance \\
\hline
\end{tabular}

each sample will be readjusted. It usually reduces the weight of samples that are classified correctly the first time and increases the weight of samples that are classified incorrectly the first time. Finally, a combination strategy is used for each weak classifier to determine the final classification result. Figure 3 is a schematic diagram of the AdaBoost algorithm.

The learning ability of FSVM [24, 25] in unbalanced samples is not good, and the AdaBoost algorithm can 
TABLE 8: User characteristics.

\begin{tabular}{lc}
\hline Feature variable & Feature name \\
\hline Size_order & Total number of products purchased by users \\
Tot_orders & Total number of user orders \\
Num_reordered & Total number of repeat purchases \\
Avg_order_size & Average number of items ordered \\
Avg_n_reordered & Average number of repurchases \\
Avg_days_between_orders & Average number of days between orders \\
Avg_order_hod & Average shopping time period \\
Avg_order_dow & Weekly purchase date \\
\hline
\end{tabular}

TABle 9: Product feature table.

\begin{tabular}{lc}
\hline Feature variable & Feature name \\
\hline Prod_orders & The total number of times the product was purchased \\
Prod_recorders & The number of times the product was repurchased \\
Prod_reorder_probability & Possibility of repurchase \\
Prod_reorder_times & Product multiple repurchases \\
Prod_reorder_ratio & Frequency of product repurchase \\
\hline
\end{tabular}

TABLE 10: User product feature table.

\begin{tabular}{lc}
\hline Feature variable & Feature name \\
\hline Avg_cart_priority & Average value of user product purchase priority \\
Times_ord & Number of purchases \\
Log_freq & Log value of user's historical purchase frequency \\
Days_to_last_order & The number of days since the most recent order \\
Order_streak & The number of other orders in the repurchase interval \\
Weekend & Weekend purchase logo \\
Hod_delta & Actual and average purchase time difference (hours) \\
Dow_delta & Actual and average purchase time difference (weeks) \\
Orders_since_last & The number of orders purchased since the last order \\
\hline
\end{tabular}

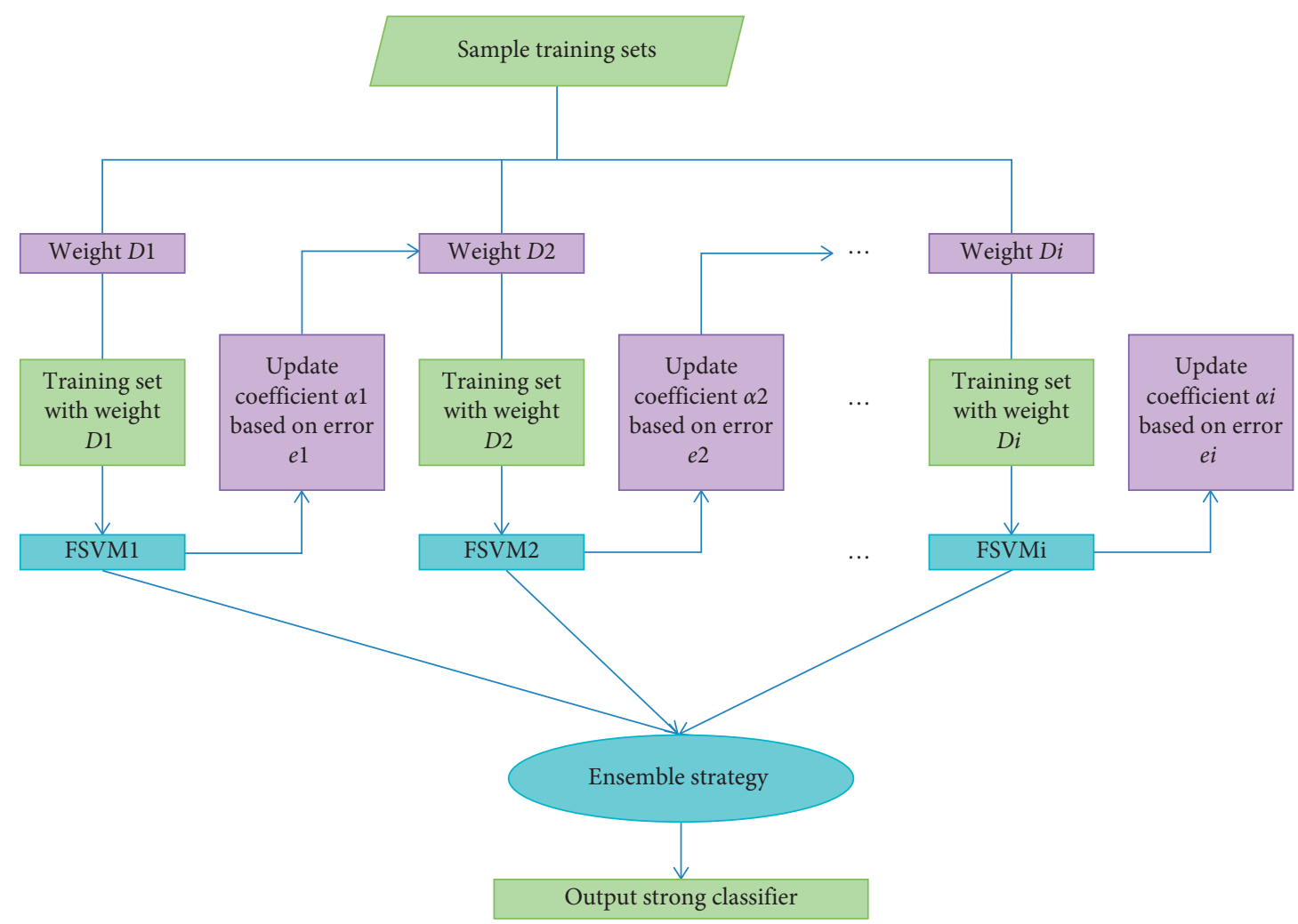

FIgURE 3: Schematic diagram of AdaBoost algorithm. 


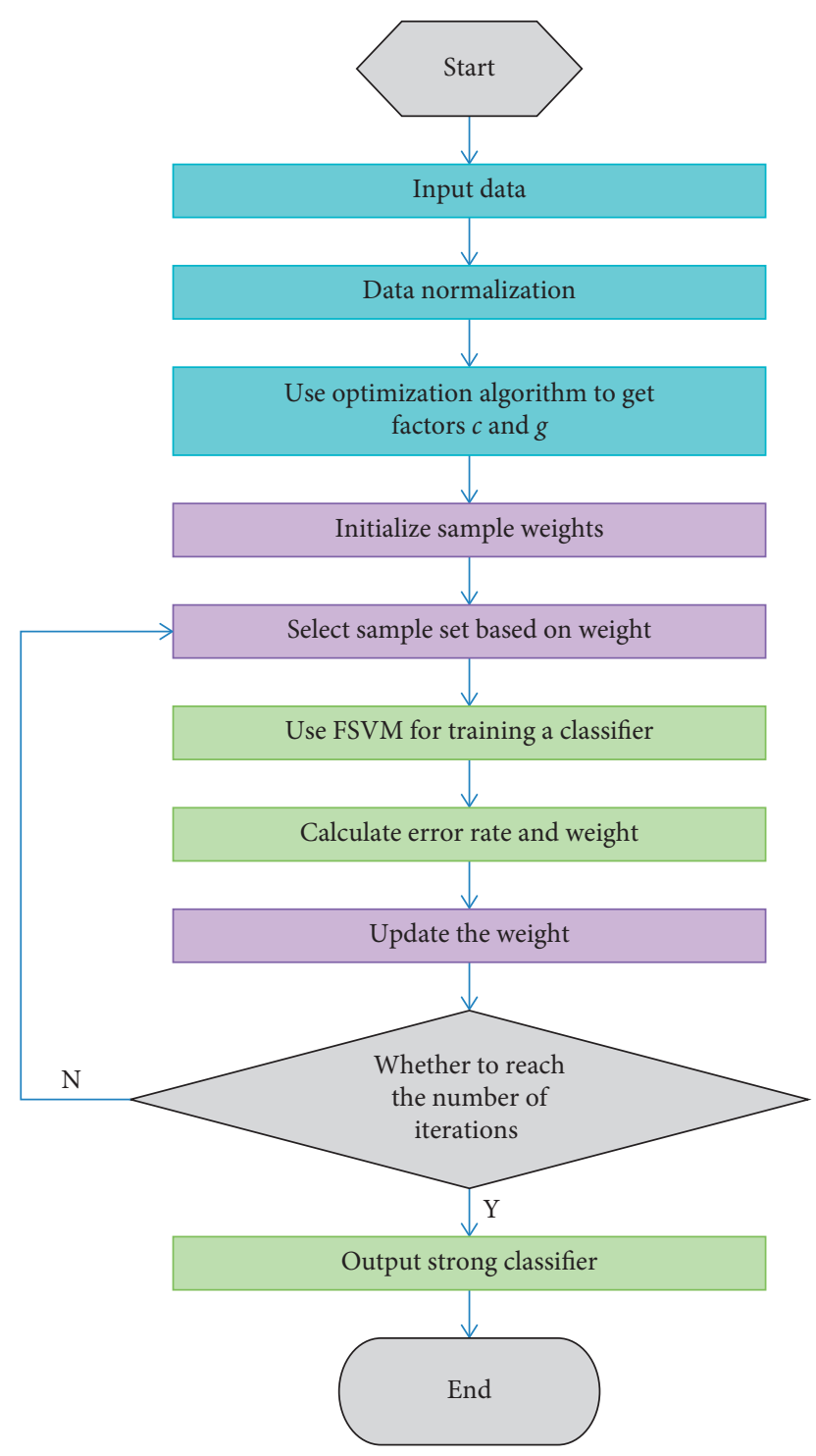

FIgUre 4: AdaBoost-FSVM algorithm flowchart.

overcome this shortcoming well, so this study proposes an AdaBoost-FSVM ensemble learning algorithm. FSVM is used as a weak classifier for ensemble learning in AdaBoost. First, the training samples are preprocessed. Second, the kernel function of SVM [26] is optimized through the grid search method to obtain the optimal penalty factor $c$ and deformation factor $g$ [27]. Initialize sample weights randomly and select a subset of training samples according to the weights of the samples. The selected sample subset is used for FSVM learning. During the learning process, different weights are assigned to the learning machine according to the training results. Update the weight of the sample according to the error rate of the training result. The sample subset used in the next round of FSVM learning is selected according to the weight of the sample. With the continuous learning of FSVM, multiple learning machines are trained. Multiple learning machines are ensembled according to the weighted voting method, and finally a strong classifier is obtained. The execution process of AdaBoost-FSVM is shown in Figure 4.
The flow of the AdaBoost-FSVM algorithm is summarized as follows.

\begin{tabular}{|c|c|}
\hline Step 1 & $\begin{array}{l}\text { The actual problem is digitized and transformed into a } \\
\text { data format that FSVM can handle }\end{array}$ \\
\hline Step & \\
\hline Step & $\begin{array}{l}\text { The grid search metho } \\
\text { parameters } c \text { an }\end{array}$ \\
\hline Step 4 & \\
\hline Step & $\begin{array}{l}\text { According to } \\
\text { selectec }\end{array}$ \\
\hline Step 6 & FSVM is used to trai \\
\hline Step & $\begin{array}{c}\text { Calculate the error } r \\
\text { weight }\end{array}$ \\
\hline & $\begin{array}{l}\text { Update the weight of } \\
\text { rate of } \mathrm{t}\end{array}$ \\
\hline Step & $\begin{array}{l}\text { etermine whether the amount of training has reached } \\
\text { the maximum number of iterations. If the maximum } \\
\text { number of iterations is not reached, go to step 6; } \\
\text { otherwise, the iteration stops. Voting is performed } \\
\text { cording to the attribute weight of the learning machine } \\
\text { generate the strongest classifier and output the result }\end{array}$ \\
\hline
\end{tabular}

3.5. Model Evaluation. After completing the model training, it is necessary to evaluate the quality of the model. The indicators for evaluating the quality of the model are shown in Table 11.

\section{Experiment and Model Evaluation}

4.1. Experimental Environment and Parameter Settings. Python language is used in this research, and PyCharm is the program running platform. Python's data analysis library pandas and graphical tool library Matplotlib are used as tools for data import, matrix transformation, and graphical display. The machine learning library scikit-learn and the algorithm framework XGBoost are used as the algorithm library for training models.

The comparison algorithms in this experiment include logistic regression (LR) [29], SVM [30], FSVM [31], random forest (RF) [32], XGBoost [33], and AdaBoost-SVM [34]. The data set used in the experiment is the original data set shown in Section 3.2. In order to study the consumer's repurchase intention of the purchased goods under the return policy, in this study, the data set was screened, and the information of consumers with return records was screened out. Among the filtered data, $70 \%$ is used as the training set, and the remaining $30 \%$ is used as the test set.

4.2. Experimental Results and Analysis. Experiments were performed on the Kaggle data set shown in Section 3.2, and the experimental results are shown in Table 12. After adding $1 \%, 3 \%$, and $5 \%$ noise data to the original data set, the experimental results obtained by each comparison algorithm are shown in Table 13.

For nonensemble algorithms, the FSVM algorithm has the best predictive effect among the four algorithms of LR, SVM, FSVM, and RF. This is because the algorithm 
TABLE 11: Model evaluation indicators.

\begin{tabular}{lcc}
\hline Index & Calculation formula & Description \\
\hline Precision ratio $(P)$ & $P=\mathrm{TP} /(\mathrm{TP}+\mathrm{FP})$ & True Positive $(\mathrm{TP})$ \\
Recall rate $(R)$ & $R=\mathrm{TP} /(\mathrm{TP}+\mathrm{FN})$ & True Negative $(\mathrm{TN})$ \\
Accuracy & Accuracy $=(\mathrm{TP}+\mathrm{TN}) /(\mathrm{P}+\mathrm{N})$ & False Positive $(\mathrm{FP})$ \\
Error rate & $\mathrm{ER}=(\mathrm{FP}+\mathrm{FN}) /(\mathrm{P}+\mathrm{N})$ & False Negative $(\mathrm{FN})$ \\
Precision & Precision $=\mathrm{TP} /(\mathrm{TP}+\mathrm{FP})$ & \\
$F 1[28]$ & $F 1=(2 \mathrm{RP} / P+N)=(2 \mathrm{TP} /($ Total number of samples $+\mathrm{TP}-\mathrm{TN}))$ & \\
\hline
\end{tabular}

The higher the accuracy, precision, and $F 1$, the better the model effect.

TABLE 12: Comparison of prediction results under different models.

\begin{tabular}{lccr}
\hline Model & Accuracy & Precision & $F 1$ \\
\hline LR & 0.7616 & 0.7178 & 0.7505 \\
SVM & 0.7704 & 0.7291 & 0.7673 \\
FSVM & 0.7912 & 0.7604 & 0.7889 \\
RF & 0.7899 & 0.7410 & 0.7782 \\
XGBoost & 0.8080 & 0.7713 & 0.7960 \\
AdaBoost-SVM & 0.8112 & 0.7698 & 0.7975 \\
AdaBoost-FSVM & 0.8493 & 0.7969 & 0.8286 \\
\hline
\end{tabular}

TABLE 13: Comparison of experimental results after adding noise.

\begin{tabular}{|c|c|c|c|c|}
\hline \multirow{2}{*}{ Noise ratio (\%) } & \multirow{2}{*}{ Algorithm } & \multicolumn{3}{|c|}{ Index } \\
\hline & & Accuracy & Precision & $F 1$ \\
\hline \multirow{7}{*}{1} & LR & 0.7436 & 0.7014 & 0.7334 \\
\hline & SVM & 0.7603 & 0.7113 & 0.7485 \\
\hline & FSVM & 0.7820 & 0.7548 & 0.7664 \\
\hline & $\mathrm{RF}$ & 0.7716 & 0.7284 & 0.7548 \\
\hline & XGBoost & 0.7837 & 0.7579 & 0.7767 \\
\hline & AdaBoost-SVM & 0.7917 & 0.7553 & 0.7798 \\
\hline & AdaBoost-FSVM & 0.8398 & 0.7910 & 0.8012 \\
\hline \multirow{7}{*}{3} & LR & 0.7408 & 0.6822 & 0.7171 \\
\hline & SVM & 0.7510 & 0.6904 & 0.7265 \\
\hline & FSVM & 0.7796 & 0.7439 & 0.7564 \\
\hline & RF & 0.7549 & 0.7045 & 0.7352 \\
\hline & XGBoost & 0.7754 & 0.7370 & 0.7565 \\
\hline & AdaBoost-SVM & 0.7801 & 0.7411 & 0.7688 \\
\hline & AdaBoost-FSVM & 0.8292 & 0.7839 & 0.7925 \\
\hline \multirow{7}{*}{5} & LR & 0.7207 & 0.6688 & 0.7010 \\
\hline & SVM & 0.7321 & 0.6719 & 0.7063 \\
\hline & FSVM & 0.7559 & 0.7359 & 0.7446 \\
\hline & RF & 0.7338 & 0.6987 & 0.7123 \\
\hline & XGBoost & 0.7530 & 0.7121 & 0.7321 \\
\hline & AdaBoost-SVM & 0.7645 & 0.7207 & 0.7448 \\
\hline & AdaBoost-FSVM & 0.8179 & 0.7764 & 0.7824 \\
\hline
\end{tabular}

introduces a fuzzy strategy to make it have better noise resistance. However, the data collected in actual production and life will inevitably carry some noise. Compared with the four algorithms of LR, SVM, RF, and FSVM, the accuracy of AdaBoost-FSVM is increased by $4 \%, 2.7 \%$, and $0.2 \%$, respectively. The precision of AdaBoost-FSVM is improved by $6 \%, 4 \%$, and $3 \%$, respectively. The $F 1$ of AdaBoost-FSVM is increased by $5 \%, 3 \%$, and $1 \%$, respectively. It can be seen from the experimental results in Table 12 that the performances of FSVM and RF are similar and that of FSVM is slightly better.
For the ensemble algorithm, the AdaBoost-FSVM algorithm among the three algorithms of XGBoost, AdaBoostSVM, and AdaBoost-FSVM has the best prediction effect. Compared with XGBoost and AdaBoost-SVM, the accuracy of AdaBoost-FSVM is increased by $5 \%$ and $5 \%$, respectively. The precision of AdaBoost-FSVM is improved by $3 \%$ and $4 \%$, respectively. AdaBoost-FSVM's $F 1$ is increased by $4 \%$ and $4 \%$, respectively. From the experimental data in Table 12, it can be seen that the prediction effects of the XGBoost and AdaBoostSVM models are similar. The accuracy, precision, and $F 1$ of AdaBoost-FSVM are improved on the basis of the two models. 
From the experimental results in Table 13, it can be analyzed that as the noise increases, the prediction performance of each algorithm decreases. When the noise increases by $1 \%$, the accuracies of LR, SVM, FSVM, RF, XGBoost, AdaBoost-SVM, and AdaBoost-FSVM are reduced by $2.3 \%, 1.3 \%, 1.2 \%, 2.3 \%, 3 \%, 2.4 \%$, and $1.1 \%$, respectively. When the noise increases by $3 \%$, the accuracies of LR, SVM, FSVM, RF, XGBoost, AdaBoostSVM, and AdaBoost-FSVM are reduced by $1.9 \%, 2.5 \%$, $4.6 \%, 4.4 \%, 4.0 \%, 3.8 \%$, and $2.3 \%$, respectively. When the noise increases by $5 \%$, the accuracies of LR, SVM, FSVM, RF, XGBoost, AdaBoost-SVM, and AdaBoost-FSVM are reduced by $5.3 \%, 4.9 \%, 4.5 \%, 7.1 \%, 6.8 \%, 5.8 \%$, and $3.7 \%$, respectively. It can be seen from the reduction rate that the AdaBoost-FSVM algorithm has the lowest reduction rate. This fully shows that the algorithm is more robust to noise.

\section{Conclusion}

The introduction of the government's return policy has prompted various e-commerce companies to launch their own return strategies and services. In order to study the impact of different return policies and services on the subsequent purchase behavior of consumers, this study selected consumer data with return records in the data set for experiments. An ensemble learning algorithm based on Boosting-FSVM is used as a predictive model. The use of ensemble learning algorithms avoids the impact of unbalanced data on the effect of the algorithm and also improves the stability of the algorithm. In addition, the base classifier in the ensemble learning algorithm is FSVM. This classification model introduces a fuzzy strategy to make the classifier have better noise resistance. To evaluate the performance of the predictive model, nonensemble and ensemble algorithms are used to predict the data set. The experimental results show that the used AdaBoost-FSVM algorithm has the best prediction effect. Using the results predicted by the AdaBoost-FSVM algorithm, e-commerce companies can more accurately analyze the return policies and services applicable to different types of consumers. This will guide e-commerce companies to adjust their return policies and services. Although the prediction performance of the prediction model used in this study has been improved to a certain extent, there is still room for further improvement. In the next step, this study will continue to study other operating methods for unbalanced data.

\section{Data Availability}

The labeled data sets used to support the findings of this study are available from the corresponding author upon request.

\section{Conflicts of Interest}

The authors declare no conflicts of interest.

\section{Acknowledgments}

This work was supported by the Scientific Research Project of Jilin Education Department.

\section{References}

[1] M. Sniegocka-Lusiewicz, "Market basket analysis in marketing research," Equilibrium, vol. 1, no. 2, pp. 115-123, 2009.

[2] S. K. Mukhopadhyay and R. Setoputro, "Reverse logistics in e-business," International Journal of Physical Distribution \& Logistics Management, vol. 34, no. 1, pp. 70-89, 2004.

[3] S. K. Mukhopadhyay and R. Setoputro, "Optimal return for e-business," PICMET-Portland International Center for Management of Engineering and Technology, vol. 2006, no. 3, pp. 1203-1209, 2006.

[4] J. D. Shulman, A. T. Coughlan, and R. C. Savaskan, "Optimal restocking fees and information provision in an integrated demand-supply model of product returns," Manufacturing \& Service Operations Management, vol. 11, no. 4, pp. 577-594, 2009.

[5] X. Su, "Consumer returns policies and supply chain performance," Manufacturing \& Service Operations Management, vol. 11, no. 4, pp. 595-612, 2009.

[6] Y. Li, X. Lei, and L. Dahui, "Examining relationships between the return policy, product quality, and pricing strategy in online direct selling," International Journal of Production Economics, vol. 144, no. 2013, pp. 451-460, 2013.

[7] J. Li, T.-M. Choi, and T. C. E. Cheng, "Mean variance analysis of fast fashion supply chains with returns policy," IEEE Transactions on Systems, Man, and Cybernetics: Systems, vol. 44, no. 4, pp. 422-434, 2014.

[8] N. Liu, T.-M. Choi, C.-W. Marcus Yuen, and N. Frankie, "Optimal pricing, modularity, and return policy under mass customization," IEEE Transactions on Systems Man \& Cybernetics Part A, vol. 42, no. 3, pp. 604-614, 2012.

[9] T.-M. Choi, C.-H. Chiu, P.-S. Chow, L. Bowood, -C. Shuk, and $\mathrm{X}$. Shen, "Mathematical modeling research in fashion and textiles supply chains and operational control systems," Mathematical Problems in Engineering, vol. 2013, no. 3, pp. 1-4, 2013.

[10] S. L. Wood, "Remote purchase environments: the influence of return policy leniency on two-stage decision processes," Journal of Marketing Research, vol. 38, no. 2, pp. 157-169, 2001.

[11] J. A. Petersen and V. Kumar, "Are product returns a necessary evil? Antecedents and consequences," Journal of Marketing, vol. 73, no. 3, pp. 35-51, 2009.

[12] S. K. Mukhopadhyay and R. Setoputro, "Optimal return policy and modular design for build-to-order products," Journal of Operations Management, vol. 23, no. 5, pp. 496506, 2005.

[13] P. Qian, F. L. Chung, S. Wang, and Z. Deng, "Fast graph-based relaxed clustering for large data sets using minimal enclosing ball," IEEE Transactions on Systems, Man, and Cybernetics, Part B (Cybernetics), vol. 42, no. 3, pp. 672-687, 2012.

[14] Y. Jiang, Z. Deng, F.-L. Chung et al., "Recognition of epileptic EEG signals using a novel multiview TSK fuzzy system," IEEE Transactions on Fuzzy Systems, vol. 25, no. 1, pp. 3-20, 2017.

[15] P. Qian, K. Zhao, Y. Jiang et al., "Knowledge-leveraged transfer fuzzy C -means for texture image segmentation with self-adaptive cluster prototype matching," Knowledge-Based Systems, vol. 130, pp. 33-50, 2017.

[16] Y. Zhang, F.-L. Chung, and S. Wang, "Clustering by transmission learning from data density to label manifold with statistical diffusion," Knowledge-Based Systems, vol. 193, p. 105330, 2020. 
[17] Y. Jiang, F. L. Chung, S. Wang, Z. Deng, J. Wang, and P. Qian, "Collaborative fuzzy clustering from multiple weighted views," IEEE Transactions on Cybernetics, vol. 45, no. 4, pp. 688-701, 2014.

[18] P. Qian, Y. Jiang, Z. Deng et al., "Cluster prototypes and fuzzy memberships jointly leveraged cross-domain maximum entropy clustering," IEEE Transactions on Cybernetics, vol. 46, no. 1, pp. 181-193, 2015.

[19] Y. Zhang, F.-L. Chung, and S. Wang, "Takagi-sugeno-kang fuzzy systems with dynamic rule weights," Journal of Intelligent \& Fuzzy Systems, vol. 37, no. 6, pp. 8535-8550, 2019.

[20] P. Qian, Y. Jiang, S. Wang et al., "Affinity and penalty jointly constrained spectral clustering with all-compatibility, flexibility, and robustness," IEEE Transactions on Neural Networks and Learning Systems, vol. 28, no. 5, pp. 1123-1138, 2016.

[21] Y. Zhang, J. Li, X. Zhou et al., "A view-reduction based multiview TSK fuzzy system and its application for textile color classification," Journal of Ambient Intelligence and Humanized Computing, pp. 1-11, 2019.

[22] L. Dery, E. Shmueli, and L. R. Boost, "BoostLR: a boostingbased learning ensemble for label ranking tasks," IEEE Access, vol. 8, pp. 176023-176032, 2020.

[23] J. R. Bertini Junior and M. D. C. Nicoletti, "An iterative boosting-based ensemble for streaming data classification," Information Fusion, vol. 45, pp. 66-78, 2019.

[24] C. F. Lin and S. D. Wang, "Fuzzy support vector machines," IEEE Transactions on Neural Networks, vol. 13, no. 3, pp. 466-471, 2002.

[25] H. P. Huang and Y. H. Liu, "Fuzzy support vector machines for pattern recognition and data mining," International Journal of Fuzzy Systems, vol. 4, no. 3, pp. 826-835, 2002.

[26] L. Christina, E. Eleazar, and S. N. William, "The spectrum kernel:a string kernel for SVM proten classification," Pacific Symposium on Biocomputing, vol. 7, pp. 566-575, 2002.

[27] P. Jonghyuck, K. Ick-Hyun, and K. Sung-Shick, "Spline regression based feature extraction for semiconductor process fault detection using support vector machine," Expert Systems with Applications, vol. 38, no. 5, pp. 5711-5718, 2010.

[28] G. Hripcsak and A. S. Rothschild, "Agreement, the F-measure, and reliability in information retrieval," Journal of the American Medical Informatics Association, vol. 12, no. 3, pp. 296-298, 2005.

[29] M. B. Naseri and G. Elliott, "A comparative analysis of artificial neural networks and logistic regression," Journal of Decision Systems, vol. 19, no. 3, pp. 291-312, 2010.

[30] S. Rogic and L. Kascelan, "Customer value prediction in direct marketing using hybrid support vector machine rule extraction method," Communications in Computer and Information Science, vol. 1064, pp. 283-294, 2019.

[31] V. Uslan, H. Seker, and R. John, "Overlapping clusters and support vector machines based interval type-2 fuzzy system for the prediction of peptide binding affinity," IEEE Access, vol. 7, pp. 49756-49764, 2019.

[32] M.-H. Roy, D. Larocque, L. ne, and Denis, "Prediction intervals with random forests," Statistical Methods in Medical Research, vol. 29, no. 1, pp. 205-229, 2020.

[33] S. Li and X. Zhang, "Research on orthopedic auxiliary classification and prediction model based on XGBoost algorithm," Neural Computing and Applications, vol. 32, no. 7, pp. 1971-1979, 2020.

[34] J. Sun, H. Li, H. Fujita, B. Fu, and W. Ai, "Class-imbalanced dynamic financial distress prediction based on adaboost-SVM ensemble combined with SMOTE and time weighting," Information Fusion, vol. 54, pp. 128-144, 2020. 